\title{
BEM ANALYSIS OF GRAVITATIONAL-CAPILLARITY WAVES ON FREE SURFACES OF COMPOUND SHELLS OF REVOLUTION
}

\author{
VASYL I. GNITKO ${ }^{1}$, ARTEM O. KARAIEV ${ }^{2 *}$, MARIA L. MYRONENKO ${ }^{1,3}$ \& ELENA A. STRELNIKOVA ${ }^{1,2 \dagger}$ \\ ${ }^{1}$ A. Podgorny Institute of Mechanical Engineering Problems of the Ukrainian Academy of Sciences, Ukraine. \\ ${ }^{2}$ V.N. Karazin Kharkiv National University, Ukraine. \\ ${ }^{3}$ O. M. Beketov National University of Urban Economy in Kharkiv, Ukraine.
}

\begin{abstract}
The paper presents a problem of gravitational-capillarity wave propagation in the frame of boundary integral equations. The wave propagation is considered in rigid compound shells of revolution. The liquid is supposed to be an ideal and incompressible one, and its flow is irrotational. The boundary value problem is formulated for Laplace's equation to obtain the velocity potential. Non-penetration boundary conditions are used at the shell's wetted surface, as well as kinematic and dynamic boundary conditions are given on the free liquid surface. Effects of surface tension are included in the Bernoulli's equation as additional pressure that is proportional to the free surface mean curvature. It allows us to consider coupled effects of both gravitational and capillarity waves. The problem is reduced to a system of singular integral equations. For their numerical simulation, the boundary element method is in use. The singular integral equations in implementation of a discrete model are transformed to linear algebraic ones, and eigenvalue problems are solved for different capillarity length numbers. Benchmark numerical investigations are presented including different kinds of compound rigid shells.
\end{abstract}

Keywords: boundary element method, gravitational-capillarity wave, rigid compound shells, singular integral equations, surface tension.

\section{INTRODUCTION}

Intense liquid sloshing in fuel tanks of spacecrafts is usually the reason of losing stability, disturbing flight trajectories, and even failure of the entire structure. Failures of first missions to Jupiter have been attributed to slosh-induced instabilities [1]. These instabilities were provoked by practically coincidence of fundamental liquid sloshing frequencies with stepping intervals of regulated units. Moreover, during the mission of spacecrafts, the different flight conditions arise, such as low gravity or overloads. So, solution of spectral problems for receiving the sloshing frequencies is very topical. Liquid sloshing under low gravity differs essentially from vibrations arising in regular or overloading gravitational fields. The issue of sloshing goes back to late 50s of the past century when lots of experimental and theoretical studies were carried out $[2,3]$. Under low gravity conditions, the surface tension of the liquid has to be taken into account. When the gravitational forces are commensurate with the surface tension, the gravity-capillary waves evolve. Such complex hydrodynamic problems arise in simulation of spacecrafts having a significant amount of liquid fuel on boards. Among the pioneering works in the area, the papers of Nayfeh [4] and Goldrick [5] need to be mentioned. The influence of surface tension on liquid behavior in the fuel tank was studied in [6] considering different Bond numbers and dynamic ratios of capillary and gravity forces. In [7], the asymptotic analysis of capillary-gravity waves under moving disturbance was accomplished. Three-dimensional statements of the problem were considered in $[8,9]$. It should be

*ORCID: https://orcid.org/0000-0003-3176-8496

†ORCID: http://orcid.org/0000-0003-0707-7214 
noted that interest to coupled gravity-capillarity interaction has notably increased in recent decades due to developing new mathematical tools together with advanced numerical methods and experimental techniques [10,11].

In this paper, the coupled problem of gravitational-capillarity wave propagation on free liquid surfaces of compound shells of revolution is formulated and solved using boundary element methods (BEMs) [12].

\section{GRAVITATIONAL-CAPILLARITY WAVE PROPAGATION ON FREE SURFACES OF COMPOUND SHELLS OF REVOLUTION}

\subsection{Problem statement}

Consider a liquid volume in equilibrium in a gravitational field and having a plane free surface. Suppose that under action of external forces, the free surface has moved from its equilibrium position. This motion will be propagated in the form of waves, which are called capillary-gravity waves [13].

The effects of gravitational-capillarity wave propagation are considered in problems of free liquid vibrations in rigid compound shells of revolution. We are concerned with rigid, partially fluid-filled shells. Designate a moistened shell surface by $S_{1}$ and the free liquid surface by $\mathrm{S}_{0}$. Suppose the Cartesian coordinate system Oxyz is connected with the shell, the liquid free surface $S_{0}$ coincides with the plane $z=h$ (Fig. 1).

We are supposed to use Euler's approach for analyzing liquid velocity distributions. The liquid is assumed to be an inviscid and incompressible one, and its flow is irrotational.

Then the velocity potential $\varphi=\varphi(x, y, z, t)$ defined as $\mathbf{v}=\nabla \varphi$ can be applied. In these assumptions, the function $\varphi$ satisfies the Laplace equation. As the liquid is located in the gravitational field, an external force acting on the dynamical system is the force of gravity. So, we have

$$
\Delta \phi=0, \quad \rho_{l} \frac{\partial \nabla \phi}{\partial t}=-\nabla P+\rho_{l} \mathbf{g} .
$$
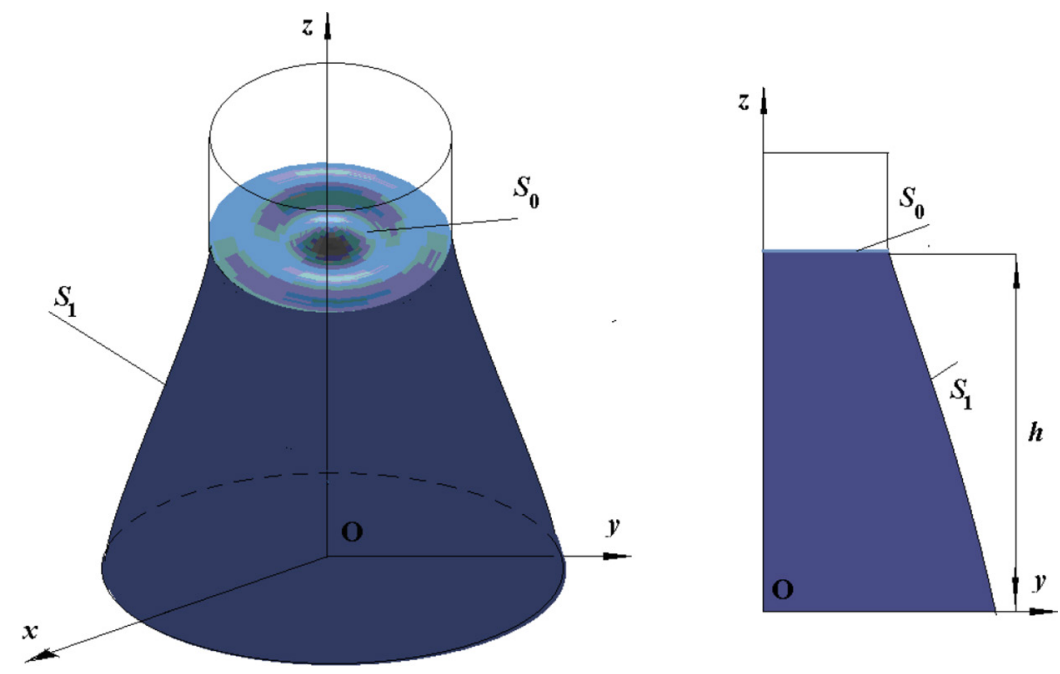

Figure 1: Compound liquid-filled shell of revolution and its draft. 
Here, $P$ is the liquid pressure, $\mathbf{g}=\mathbf{e}_{\mathbf{z}} g$ is the gravity acceleration, and $\rho_{l}$ is for the liquid density.

Equation (1) can be modified into Bernoulli's equation

$$
\frac{\partial \varphi}{\partial t}+\frac{1}{\rho_{l}} P-(\mathbf{g}, \mathbf{r})=C(t)
$$

where $\mathbf{r}$ is the radius vector of points inside the liquid domain and $C(t)$ is an unknown time-dependant function.

Now let us find the representation of eqn (2) on the free surface. The pressure on the free surface consists of atmospheric pressure $P_{0}$ and additional capillarity pressure, which can be explained as an effect of a surface tension

$$
P=P_{0}+2 \sigma H
$$

where $H$ is the mean curvature of the free surface and s is the surface tension.

Time-dependant function $C(t)$ can be added into the potential, so the linear presentation of Bernoulli's equation on the free surface is as follows:

$$
\frac{\partial \varphi}{\partial t}+g \zeta-\frac{2 \sigma}{\rho_{l}} H=0
$$

where $\zeta=\zeta(x, y, t)$ is a function describing the free surface profile.

In general case, the mean curvature can be expressed through the divergence of the unit normal vector to the free surface

$$
\left.\mathbf{n}\right|_{S_{0}}=\left(-\frac{\partial \zeta}{\partial \rho}, 1\right) / \sqrt{1+\zeta_{\rho}^{\prime 2}}
$$

by the following expression:

$$
H=-\frac{1}{2} \operatorname{divn}=\frac{1}{2 \rho} \frac{\partial}{\partial \rho}\left[\rho \frac{\partial \zeta}{\partial \rho}\left(1+\left(\frac{\partial \zeta}{\partial \rho}\right)^{2}\right)^{-\frac{1}{2}}\right]
$$

In linear approximation, this equation simplifies into the Laplacian of the free surface profile on the plane perpendicular to the $\mathrm{O} z$ axis

$$
H=\frac{1}{2} \Delta \zeta
$$

So, using eqn (7), we get the linear form of Bernoulli's equation as follows:

$$
\frac{1}{g} \frac{\partial \varphi}{\partial t}+\zeta-\lambda_{c}^{2} \Delta \zeta=0
$$

where $\lambda_{c}=\sqrt{\frac{\sigma}{\rho_{l} g}}$ is the capillarity length [13].

To get the closed system for obtaining the unknown potential, we must include the relationship between Euler's and Lagrange's velocities on the free surface [14]: 


$$
\frac{\partial \zeta}{\partial t}=\frac{\partial \varphi}{\partial n}
$$

Taking the time derivative of eqn (8) and using condition (9), we have

$$
\frac{1}{g} \frac{\partial^{2} \varphi}{\partial t^{2}}+\frac{\partial \varphi}{\partial n}-\lambda_{c}^{2} \Delta_{p} \frac{\partial \varphi}{\partial n}=0
$$

Operator $\Delta_{p}$ here is Laplacian in the plane perpendicular to $\mathrm{O} z$ axis. It can be expressed through the standard Laplacian by the following formula:

$$
\Delta_{p}=\Delta-\left(\mathbf{e}_{\mathbf{z}}\left(\mathbf{e}_{\mathbf{z}}, \nabla\right), \nabla\right) .
$$

On the side walls and bottom of the shell, the velocity normal component is equal to zero, so the boundary condition can be expressed in the next form:

$$
\left.\frac{\partial \varphi}{\partial n}\right|_{S_{1}}=0
$$

Equations (1), (10), and (12) make up the following closed system for receiving the potential:

$$
\Delta \varphi=0, \quad \frac{1}{g} \frac{\partial^{2} \varphi}{\partial t^{2}}+\frac{\partial \varphi}{\partial n}-\left.\lambda_{c}^{2} \Delta \Delta_{p} \frac{\partial \varphi}{\partial n}\right|_{S_{0}}=0,\left.\quad \frac{\partial \varphi}{\partial n}\right|_{S_{1}}=0 .
$$

The solvability condition is needed to be added [15]

$$
\int_{S_{0}} \frac{\partial \phi}{\partial n} d S_{0}=0
$$

Solution of eqns(13) and (14) allows us to receive the potential $\mathrm{j}$ that describes both gravitational and capillarity effects on the free surface of the liquid in partially fluid-filled shells.

\subsection{Gravitational stationary states expansion}

Here, we propose to represent the potential function for describing the coupled capillarygravity wave propagation as the following expansion of gravitational stationary states:

$$
\varphi(\mathbf{r}, t)=\sum_{k=1}^{\infty} \alpha_{k} \psi_{k}^{(g)}(\mathbf{r}) e^{i \omega_{k} t},
$$

where $\alpha_{k}$ is an unknown constant, $\omega_{k}$ an unknown liquid oscillation frequency, and $\psi_{k}^{(g)}(\mathbf{r})$ is the stationary state of the only gravitational field, without including the effects of surface tension. Functions $\psi_{k}^{(g)}(\mathbf{r}), \quad k=1,2, \ldots$ satisfy the following relations:

$$
\Delta \psi_{k}^{(g)}=0,\left.\quad \frac{\partial \psi_{k}^{(g)}}{\partial n}\right|_{S_{0}}=\frac{\omega_{k}^{(g) 2}}{g} \psi_{k}^{(g)},\left.\quad \frac{\partial \psi_{k}^{(g)}}{\partial n}\right|_{S_{1}}=0 .
$$

Here, $\omega_{k}^{(g)}$ is the frequency of liquid vibrations in the regular or overloading gravitational field with the acceleration gravity $g$. 
The solvability condition takes the form

$$
\int_{S_{0}} \frac{\partial \psi_{k}^{(g)}}{\partial n} d S_{0}=0
$$

To find the relationship between the only gravitational frequencies and coupled gravitational-capillarity ones, we substitute eqn (15) into boundary condition on the free surface, namely, in the second equation in eqn (13) and get

$$
\left.\sum_{k=1}^{\infty} \alpha_{k}\left[-\frac{\omega_{k}^{2}}{g} \psi_{k}^{(g)}+\frac{\omega_{k}^{(g) 2}}{g} \psi_{k}^{(g)}-\lambda_{c}^{2} \frac{\omega_{k}^{(g) 2}}{g} \Delta_{p} \psi_{k}^{(g)}\right] e^{i \omega_{k} t}\right|_{S_{0}}=0 .
$$

Taking the dot product of linear combinations in eqn (18) and an arbitrary gravitational function $\psi_{l}^{(g)} l=1,2, \ldots$, and using the orthogonal property of eigenvectors, one can obtain the next relation:

$$
\alpha_{l} \frac{\omega_{l}^{2}}{g} \psi_{l}^{(g)} e^{i \omega_{l} t}=\alpha_{l} \frac{\omega_{l}^{(g) 2}}{g} \psi_{l}^{(g)} e^{i \omega_{l} t}-\lambda_{c}^{2} \sum_{k=1}^{\infty} \alpha_{k} \frac{\omega_{k}^{(g) 2}}{g}\left(\Delta_{p} \psi_{k}^{(g)}, \psi_{l}^{(g)}\right) e^{i \omega_{k} t}
$$

As the unperturbed state of free surface is assumed to be the plane, we can easily calculate plane Laplacian $\Delta_{p}$ of the gravitational state function $\psi_{k}^{(g)}$

$$
\Delta_{p} \psi_{k}^{(g)}=-\frac{\partial^{2} \psi_{k}^{(g)}}{\partial z^{2}}=-\frac{\omega_{k}^{(g) 2}}{g} \frac{\partial \psi_{k}^{(g)}}{\partial z}=-\frac{\omega_{k}^{(g) 4}}{g^{2}} \psi_{k}^{(g)} .
$$

Final relationship between the only gravitational frequencies and coupled gravitationalcapillarity frequencies is obtained as follows:

$$
\frac{\omega_{l}^{2}}{g}=\frac{\omega_{l}^{(g) 2}}{g}\left[1+\lambda_{c}^{2} \frac{\omega_{l}^{(g) 4}}{g^{2}}\right], \quad l=1,2, \ldots
$$

So, for receiving the frequencies of coupled gravitational-capillarity waves propagation, it is necessary to find the gravitational state functions $\psi_{k}^{(g)}$ and frequencies $\omega_{k}^{(g)}$ of liquid vibrations in the regular or overloading gravitational fields and use eqns (15) and (21).

\section{NUMERICAL SIMULATION OF GRAVITATIONAL STATIONARY STATES AND FREQUENCIES}

\subsection{Implementation of BEM}

To define the functions $\psi_{k}^{(g)}$ and frequencies $\omega_{k}^{(g)}$, the BEM in its direct formulation [12] is in use. Dropping indices $\underset{k}{(g)}$, one can obtain the following integral equation:

$$
2 \pi \psi\left(\mathbf{r}_{0}\right)=\iint_{S} q \frac{1}{\left|\mathbf{r}-\mathbf{r}_{0}\right|} d S-\iint_{S} \psi \frac{\partial}{\partial n} \frac{1}{\left|\mathbf{r}-\mathbf{r}_{0}\right|} d S
$$


Here, $S=S_{1} \cup S_{0}$ and points $\mathbf{r}$ and $\mathbf{r}_{0}$ belong to the surface $S$. The value $\left|\mathbf{r}-\mathbf{r}_{0}\right|$ is the Cartesian distance between $\mathbf{r}$ and $\mathbf{r}_{0}$ and $q=\partial \psi / \partial n$ is a flux. Let us denote by $\psi_{1}$ the values of $\psi$ on the wetted surface $S_{1}$ and by $\psi_{0}$ the values of $\psi$ on the free surface $S_{0}$.

With BEM formulation, the following system of singular integral equations is obtained from boundary conditions (16):

$$
\begin{gathered}
2 \pi \psi_{1}+\iint_{S_{1}} \psi_{1} \frac{\partial}{\partial n} \frac{1}{\left|\mathbf{r}-\mathbf{r}_{0}\right|} d S_{1}-\frac{\omega^{2}}{g} \iint_{S_{0}} \psi_{0} \frac{1}{\left|\mathbf{r}-\mathbf{r}_{0}\right|} d S_{0}+\iint_{S_{0}} \psi_{0} \frac{\partial}{\partial n} \frac{1}{\left|\mathbf{r}-\mathbf{r}_{0}\right|} d S_{0}=0, \quad \mathbf{r}_{0} \in S_{1}, \\
\quad-\iint_{S_{1}} \psi_{1} \frac{\partial}{\partial n} \frac{1}{\mathbf{r}-\mathbf{r}_{0}} d S_{1}-2 \pi \psi_{0}+\frac{\omega^{2}}{g} \iint_{S_{0}} \psi_{0} \frac{1}{\mathbf{r}-\mathbf{r}_{0}} d S_{0}=0, \mathbf{r}_{0} \in S_{0} .
\end{gathered}
$$

Introduce the following integral operators:

$$
\mathbf{R}\left(\sigma_{1}, \sigma_{2}\right) \psi=\iint_{\sigma_{1}} \psi \frac{\partial}{\partial n} \frac{1}{\left|\mathbf{r}-\mathbf{r}_{0}\right|} d \sigma_{1}, \mathbf{Q}\left(\sigma_{1}, \sigma_{2}\right) \psi=\iint_{\sigma_{1}} \psi \frac{1}{\left|\mathbf{r}-\mathbf{r}_{0}\right|} d \sigma_{1}, \mathbf{r} \in \sigma_{1}, \mathbf{r}_{0} \in \sigma_{2}
$$

when $\sigma_{1}=\sigma_{2}=\sigma$, we obtain

$$
\mathbf{R}(\sigma, \sigma) \psi=2 \pi \mathbf{I} \psi+\iint_{\sigma} \psi \frac{\partial}{\partial n} \frac{1}{\left|P-P_{0}\right|} d \sigma
$$

where $\mathbf{I}$ is the identity tensor.

Then eqn (23) can be written as

$$
\begin{gathered}
\mathbf{R}\left(S_{1}, S_{1}\right) \psi_{1}-\frac{\omega^{2}}{g} \mathbf{Q}\left(S_{0}, S_{1}\right) \psi_{0}+\mathbf{R}\left(S_{0}, S_{1}\right) \psi_{1}=0, \\
-\mathbf{R}\left(S_{1}, S_{0}\right) \psi_{1}-2 \pi \mathbf{I} \psi_{0}+\frac{\omega^{2}}{g} \mathbf{Q}\left(S_{0}, S_{0}\right) \psi_{0}=0 .
\end{gathered}
$$

Considering the shells of revolution, replace Cartesian coordinates $(x, y, z)$ with cylindrical ones $(r, \mathrm{q}, z)$

$$
x=\rho \cos \theta ; \quad y=\rho \sin \theta ; \quad z=z
$$

and perform integration in eqn (26) with respect to the circumference coordinate $\mathrm{q}$ as in [16], taking into account that

$$
\mathbf{r}=(\rho, \theta, z), \mathbf{r}_{0}=\left(\rho_{0}, \theta_{0}, z_{0}\right),\left|\mathbf{r}-\mathbf{r}_{0}\right|=\sqrt{\rho^{2}+\rho_{0}^{2}+\left(z-z_{0}\right)^{2}-2 \rho{ }_{0} \cos \left(\theta-\theta_{0}\right)} .
$$

Then the integral operators $\mathbf{R}$ and $\mathbf{Q}$ can be expressed on terms of complete elliptic integrals as follows [10]:

$$
\mathbf{R}(\sigma, \sigma)=2 \pi \mathbf{I}+A_{1}\left(\mathbf{r}, \mathbf{r}_{0}\right) \mathrm{E}(k)+A_{2}\left(\mathbf{r}, \mathbf{r}_{0}\right) \mathrm{K}(k) ; \quad \mathbf{Q}(\sigma, \sigma)=B\left(\mathbf{r}, \mathbf{r}_{0}\right) \mathrm{K}(k) .
$$

Here, 


$$
\begin{aligned}
& A_{1}\left(\mathbf{r}, \mathbf{r}_{0}\right)=\frac{4}{\sqrt{c} d}\left[\frac{\rho^{2}-\rho_{0}^{2}+\hat{z}^{2}}{2 \rho} n_{r}+\hat{z} n_{z}\right] A_{2}\left(\mathbf{r}, \mathbf{r}_{0}\right)=-\frac{2 n_{r}}{\rho \sqrt{c}} \quad B\left(\mathbf{r}, \mathbf{r}_{0}\right)=\frac{4}{\rho \sqrt{c}}, \\
& \hat{z}=z_{0}-z, \quad a=\rho^{2}+\rho_{0}^{2}+\hat{z}^{2}, \quad b=2 \rho_{0}, \quad c=a+b, \quad d=a-b, \quad k^{2}=\frac{2 b}{a+b},
\end{aligned}
$$

$n_{r}, n_{z}$ are the components of the external unit normal $\mathbf{n}$ to the surface $\sigma$ in the $\rho$ and $z$ directions, respectively, and $\mathrm{E}(k), \mathrm{K}(k)$ are complete elliptic integrals

$$
\mathrm{E}(k)=\int_{0}^{\pi / 2}\left(1-k^{2} \sin ^{2} \theta\right)^{1 / 2} d \theta, \quad \mathrm{K}(k)=\int_{0}^{\pi / 2}\left(1-k^{2} \sin ^{2} \theta\right)^{-1 / 2} d \theta .
$$

After excluding function $\psi_{1}$ from eqn (26), the following eigenvalue problem is formulated:

$$
\left(\mathbf{D A} \mathbf{A}^{-1} \mathbf{C}+\mathbf{I}\right) \psi_{0}-\lambda\left(\mathbf{D A} \mathbf{A}^{-1} \mathbf{B}+\mathbf{F}\right) \psi_{0}=0 ; \quad \lambda=\omega^{2} / g
$$

where

$$
\mathbf{A}=\mathbf{R}\left(S_{1}, S_{1}\right), \mathbf{B}=\mathbf{Q}\left(S_{0}, S_{1}\right), \mathbf{C}=\mathbf{R}\left(S_{0}, S_{1}\right), \mathbf{D}=-\mathbf{R}\left(S_{1}, S_{0}\right), \mathbf{F}=\mathbf{Q}\left(S_{0}, S_{0}\right) \psi_{0}=0
$$

Diagonal elements of matrices in eqn (32) are singular integrals; for their calculation, we use the technique described in [17].

Solution of problem in eqn (31) gives the gravitational state functions $\psi_{k}^{(g)}$ and frequencies $\omega_{k}^{(g)}$ for liquid sloshing in the rigid compound shell of revolution.

\subsection{Validation of the proposed method}

The axisymmetrical gravitational state functions and frequencies are obtained numerically for cylindrical (Fig. 2a), conical (Fig. 2b), and spherical (Fig. 2c) shells in the regular gravitational field and considering coupled gravity-capillarity effects. For all considered shells, the filling level is denoted as $h$.

The numerical solutions are obtained by using the BEM described previously. In the present numerical simulation for cylindrical and conical shells, we used 100 boundary elements along the bottom, wetted cylindrical or conical parts and 100 elements along the radius of free surface. For the spherical shell, 200 elements along the spherical surface and 150 elements along the free surface were applied. A further increase in the number of elements did not lead to essential changes in the results.

The circular cylindrical shell with a flat bottom (Fig. 2d) is considered with the following parameters: radius of cylinder is $R=1 \mathrm{~m}$ and the filling level is $h=2 \mathrm{~m}$.

For testifying the proposed numerical algorithm, the analytical solution of Ibrahim [18] for frequencies and modes of cylindrical shell is in use:

$$
\frac{\omega_{k}^{(g) 2}}{g}=\frac{\mu_{k}}{R} \tanh \left(\mu_{k} \frac{h}{R}\right), k=1,2, . . ; \psi_{k}^{(g)}=\mathrm{J}_{0}\left(\frac{\mu_{k}}{R} r\right) \cosh \left(\frac{\mu_{k}}{R} z\right) \cosh ^{-1}\left(\frac{\mu_{k}}{R} h\right) .
$$

Here, $\mu_{k}$ is the root of the equation $\mathrm{J}_{0}^{\prime}(x)=0$ and $\mathrm{J}_{0}(x)$ is Bessel function of the first kind.

Capillarity effects correct the frequencies formulae by adding the surface tension term 


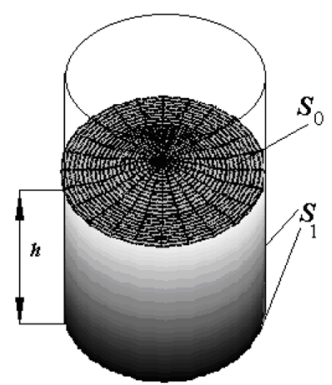

(a)

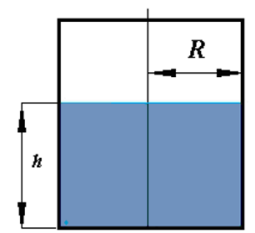

(d)

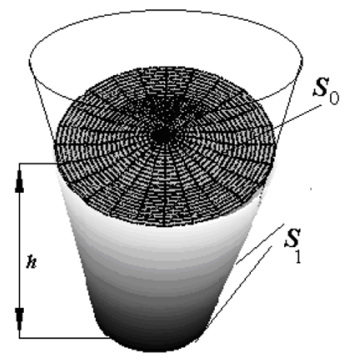

(b)

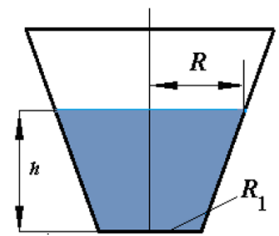

(e)

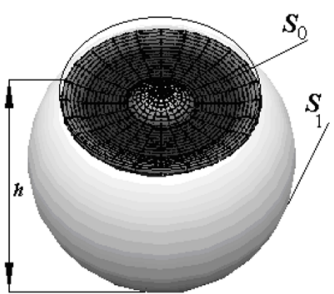

(c)

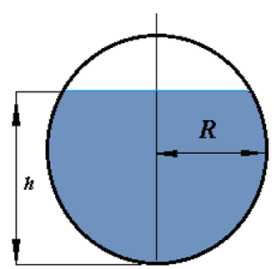

(f)

Figure 2: Partially liquid-filled shells of revolution.

$$
\frac{\omega_{k}^{2}}{g}=\left(\frac{\mu_{k}}{R}+\lambda_{c}^{2} \frac{\mu_{k}^{3}}{R^{3}}\right) \tanh \left(\mu_{k} \frac{h}{R}\right), k=1,2, \ldots
$$

Table 1 provides the numerical and analytical values of the frequency parameter $\omega_{k}^{2} / g$ for different mode numbers $k$, with capillarity length $\lambda_{c}^{2}=10^{-3}$.

Next, we consider the liquid sloshing in conical tanks. The following parameters of conical tanks are chosen: free surface radius is $R=1 \mathrm{~m}$ and the filling level is $h=1 \mathrm{~m}$. Different values of $R_{1}$ are considered (Fig. 2e).

In Table 2, the results of numerical simulation are presented for different values of $R_{1}$. Comparison of results obtained by the proposed method without capillarity effects with the data of Gavrilyuk et al. [19] is presented. In [19], the semi-analytical method was proposed. The results obtained by both methods are in good agreement.

In Table 3, the frequencies corresponding to the first mode axisymmetric vibrations considering capillarity effects with $\lambda_{c}^{2}=10^{-3}$ for liquid sloshing in conical tanks are given.

Table 1: Comparison of analytical and numerical results.

\begin{tabular}{llllll}
\hline \multirow{1}{*}{ Method } & \multicolumn{5}{c}{ Frequency parameter $\omega_{k}^{2} / g$} \\
& & \multicolumn{4}{c}{$\begin{array}{c}\text { Mode number } k \\
\end{array}$} \\
& $\mathbf{1}$ & $\mathbf{2}$ & $\mathbf{3}$ & $\mathbf{4}$ & $\mathbf{5}$ \\
\hline Proposed BEM & 3.884 & 7.365 & 10.235 & 15.703 & 20.956 \\
Exact solution & 3.888 & 7.361 & 11.226 & 15.689 & 20.939 \\
\hline
\end{tabular}


Table 2: Frequency parameters for conical tanks without capillarity effects.

\begin{tabular}{llllll}
\hline \multirow{2}{*}{ Method } & \multicolumn{5}{c}{ Frequency parameter $\omega_{k}^{(g) 2} / g$} \\
& $\mathbf{0 . 8}$ & $\mathbf{0 . 6}$ & $\mathbf{0 . 4}$ & $\mathbf{0 . 2}$ & $\mathbf{0 . 1}$ \\
\hline$[19]$ & 3.386 & 3.386 & 3.382 & 3.139 & 2.187 \\
BEM & 3.389 & 3.390 & 3.391 & 3.192 & 2.200 \\
\hline
\end{tabular}

Table 3: Frequency parameters for conical tanks with capillarity effects.

\begin{tabular}{lllll}
\hline \multicolumn{5}{c}{ Frequency parameter $\omega_{k}^{(g) 2} / g$} \\
& $R_{\mathbf{1}}, \mathbf{m}$ \\
\hline 0.8 & 0.6 & 0.4 & 0.2 & 0.1 \\
3.428 & 3.429 & 3.430 & 3.225 & 2.211 \\
\hline
\end{tabular}

The sloshing frequencies of the spherical shell of radius $R=1 \mathrm{~m}$ partially filled with the ideal incompressible fluid upon different filling levels $h$ are estimated. Numerical analysis is carried out for $0.2<h / R<1.9$. It would be noted that value $h=1.99 \mathrm{~m}$ corresponds to the so-called "ice-fishing" problem, where formally, an infinitely wide and deep ocean covered with ice, with a small round fishing hole is under consideration. Sloshing in such “containers" was studied by McIver [20]. Comparison of the results is presented in Table 4.

Table 5 shows the frequencies corresponding to the first two modes of axisymmetric vibrations considering the capillarity effects with $\lambda_{c}^{2}=10^{-3}$ for liquid sloshing in spherical shells.

Table 4: Frequency of axisymmetric liquid oscillations in the fluid-filled spherical shell.

\begin{tabular}{|c|c|c|c|c|c|c|}
\hline \multirow[t]{2}{*}{$k$} & \multirow[t]{2}{*}{ Method } & \multicolumn{5}{|c|}{$\begin{array}{c}\text { Frequency parameter } \omega_{k}^{(g) 2} / g \\
\text { Filling level } h, \mathbf{m}\end{array}$} \\
\hline & & 0.2 & 0.6 & 1.0 & 1.8 & 1.99 \\
\hline \multirow[t]{2}{*}{1} & [20] & 3.8261 & 3.6501 & 3.7451 & 6.7641 & 29.2151 \\
\hline & BEM & 3.8314 & 3.6510 & 3.7456 & 6.7665 & 29.1811 \\
\hline \multirow[t]{2}{*}{2} & [20] & 9.2561 & 7.2659 & 6.9763 & 12.1139 & 52.0467 \\
\hline & BEM & 9.2686 & 7.2684 & 6.9780 & 12.1205 & 52.0255 \\
\hline
\end{tabular}

Table 5: Frequency of axisymmetric liquid oscillations in the fluid-filled spherical shell.

\begin{tabular}{cccccrc}
\hline & & \multicolumn{5}{c}{ Frequency parameter $\omega_{k}^{(g) 2} / g$} \\
\multicolumn{1}{c}{$k$} & Method & \multicolumn{5}{c}{ Filling level $h, \mathbf{m}$} \\
& & $\mathbf{0 . 2}$ & $\mathbf{0 . 6}$ & $\mathbf{1 . 0}$ & $\mathbf{1 . 8}$ & $\mathbf{1 . 9 9}$ \\
\hline 1 & BEM & 3.4201 & 3.6997 & 3.7981 & 7.0763 & 54.0299 \\
2 & BEM & 10.0648 & 7.6524 & 7.3178 & 13.9011 & 192.8405 \\
\hline
\end{tabular}


From the results shown in Tables 1,3 , and 5 , it is possible to make a conclusion that the capillarity effects have essential influence on the frequency parameters. Even with $\lambda_{c}^{2}=10^{-3}$, one can see changes in the parameter $\omega_{k}^{(g) 2} / g$ by at least $10 \%$.

The results of numerical simulation demonstrate high accuracy and efficiency of the proposed BEM and reveal the new possibility of considering the effects of surface tension on the liquid vibration frequencies. The mode shapes are Bessel like for all considered shells (Fig. $2 \mathrm{a}-\mathrm{c})$.

\section{NUMERICAL SIMULATION OF AXISYMMRTRIC LIQUID-FREE VIBRATIONS IN COMPOUND SHELLS}

Free axisymmetric harmonic liquid vibrations in the fluid-filled rigid compound shell are examined. The cylindrical (CS), cylindrical-conical (CCS), and compound cylindricalspherical (CSS) shells are considered (Fig. 3a-c).

Denote the radii of the cylindrical and spherical parts by $R$, height of the wetted part by $h$, height of cylindrical part in compound shells by $h_{1}$ (Fig. $3 \mathrm{~b}$ and c), and height and radius of the conical part by $h_{2}$ and $R_{1}$ (Fig. 3b).

As before, suppose that the shells are related to the Cartesian coordinate system Oxyz, and the liquid-free surface $S_{0}$ coincides with the plane $z=h$ at the state of rest. The axisymmetric free liquid vibrations in three rigid shells depicted in Fig. 3 are studied. The fluid-filling level $h$ and radius $R$ of the cylindrical parts are equal for all considered shells. In the following numerical simulation, the values $R=h_{1}=h_{2}=1 \mathrm{~m}, R_{1}=0.5 \mathrm{~m}$, and $h=2 \mathrm{~m}$ are assigned (Fig. 3 ). The requisite number of boundary elements for evaluating unknown functions with given accuracy was estimated by the authors in [14]. It was shown that applying 180 boundary elements along the wetted parts of shells and 100 elements along the free surface radius is sufficient for reaching accuracy e $=10^{-3}$.

Good agreement between the analytical results for the frequencies of CS obtained by Ibrahim [18] and the numerical ones received by proposed axisymmetric BEM is presented in Table 6.

Analyzing the results of Table 6, one can observe that the sloshing frequencies of cylindrical, cylindrical-spherical, and cylindrical-spherical shells with equal filling levels are very close. So, for approximate estimation of these frequencies in compound shells of revolution with different forms of bottoms (Fig. 3b, c), one can use the circular cylindrical shell with the flat bottom (Fig. 3a), supposing equal filling levels $h$ for all the shells.

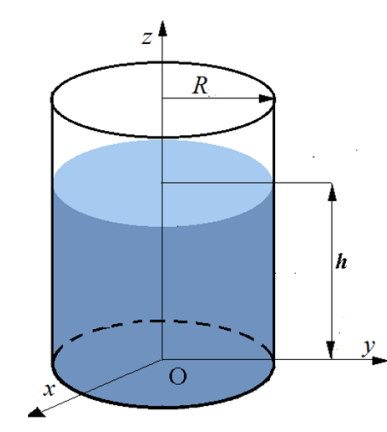

(a)

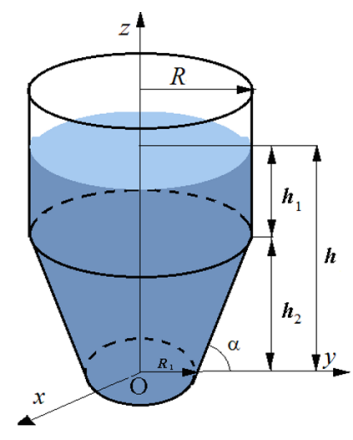

(b)

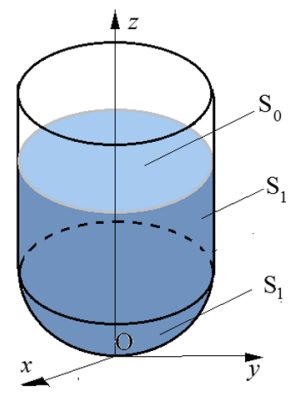

(c)

Figure 3: Liquid-filled shells with different bottoms. 
Table 6: Frequencies of axisymmetric liquid vibrations in different shells.

\begin{tabular}{lccccccc}
\hline $\begin{array}{c}\text { Type of shell and } \\
\text { method }\end{array}$ & \multicolumn{7}{c}{$\begin{array}{c}\text { Frequency parameter } \omega_{k}^{2} / g \\
\text { Mode number } k\end{array}$} \\
& $\mathbf{1}$ & $\mathbf{2}$ & $\mathbf{3}$ & $\mathbf{4}$ & $\mathbf{5}$ & $\mathbf{6}$ & $\mathbf{7}$ \\
\hline CS, BEM & 3.827 & 7.010 & 10.165 & 13.345 & 16.488 & 19.700 & 22.874 \\
CS, analytical & 3.827 & 7.009 & 10.163 & 13.340 & 16.480 & 19.695 & 22.857 \\
solution & & & & & & & \\
CCS, BEM & 3.827 & 7.010 & 10.166 & 13.343 & 16.488 & 19.709 & 22.874 \\
CSS, BEM & 3.827 & 7.011 & 10.168 & 13.343 & 16.488 & 19.709 & 22.874 \\
\hline
\end{tabular}

Table 7: Frequency of axisymmetric liquid oscillations in compound shells.

\begin{tabular}{lccccccc}
\hline $\begin{array}{c}\text { Type of shell and } \\
\text { method }\end{array}$ & $\mathbf{1}$ & $\mathbf{7}$ & \multicolumn{7}{c}{ Frequency parameter $\omega_{k}^{2} / g$} \\
& Mode number $k$ \\
& $\mathbf{1}$ & $\mathbf{2}$ & $\mathbf{3}$ & $\mathbf{4}$ & $\mathbf{5}$ & $\mathbf{6}$ & $\mathbf{7}$ \\
\hline CS, BEM & 3.883 & 7.355 & 11.215 & 15.722 & 20.970 & 27.345 & 34.842 \\
CCS, BEM & 3.883 & 7.355 & 11.217 & 15.719 & 20.970 & 27.365 & 34.842 \\
CSS, BEM & 3.883 & 7.356 & 11.217 & 15.719 & 20.970 & 27.365 & 34.842 \\
\hline
\end{tabular}

Table 7 shows the frequencies corresponding to the first modes of axisymmetric vibrations of liquid in compound shells of revolution, considering capillarity effects with $\lambda_{c}^{2}=10^{-3}$.

Results of Table 7 show increase in the frequency parameter when the capillarity effects are taken into account, and it should be pointed out that the higher the frequency number, the greater the change.

\section{CONCLUSION}

The BEM is extended to the problems of gravitational-capillarity wave propagation on free surfaces of compound shells of revolution. The three-dimensional axisymmetric problems of simulating the liquid vibrations are reduced to one-dimensional systems of singular integral equations. The kernels of integral operators in the obtained systems are expressed in terms of complete elliptic integrals. Benchmark numerical investigations are presented, including the cylindrical, conical, and spherical shells. The comparative analysis of liquid vibrations in cylindrical, cylindrical-spherical, and cylindrical-spherical shells is accomplished.

\section{ACKNOWLEDGEMENTS}

Financial support for the project from the Ministry of Education and Science of Ukraine is gratefully acknowledged. The authors would also like to thank their foreign collaborator, Professor Alexander Cheng, University of Mississippi, USA, for his constant support and interest in the research. 


\section{REFERENCES}

[1] O’Neil, W.J., Project Galileo - The Jupiter Mission. In Barbieri C., Rahe J.H., Johnson T.V. \& Sohus A.M., eds., The Three Galileos: The Man, the Spacecraft, the Telescope. Astrophysics and Space Science Library, Springer, Dordrecht. 1997, 220, pp. 1-6.

[2] Silveira, A.M., Stephens, D.G., Leonard, H.W., An Experimental Investigation of Liquid Oscillation in Tanks with Various Baffles, NASA Technical No te D-715, pp. 5-24, 1961.

[3] Abramson, H.N., Slosh Suppression, NASA Technical Report SP-8031, pp. 27-40, 1969.

[4] Nayfeh, Ali Hasan., Third harmonic resonance in the interaction of capillary and gravity weaves. Journal of Fluid Mechanics, 48(2), pp. 385-395, 1971. https://doi.org/10.1017/ s0022112071001630

[5] Goldrick, L. F. Mc., An experiment on second-order capillary gravity resonant wave interaction. Journal of Fluid Mechanics, 40(2), pp. 251-271, 1970. https://doi.org/ $10.1017 / \mathrm{s} 0022112070000162$

[6] Hung, R.J., Lee, C.C. \& Leslie F.W., Similarity rules in gravity jitterrelated spacecraft liquid propellant slosh waves excitation. Journal of Fluids and Structures, 6(4), pp. 493-522, 1992. https://doi.org/10.1016/0889-9746(92)90028-2

[7] Liang, H. \& Chen, X., Asymptotic analysis of capillary-gravity waves generated by a moving disturbance. European Journal of Mechanics - B/Fluids, 72, pp. 624-630, 2018. https://doi.org/10.1016/j.euromechflu.2018.08.012

[8] Milewski, P.A., Three-dimensional localized solitary gravity-capillary waves. Comm. Math., 3, 89-99, 2005. https://doi.org/10.4310/cms.2005.v3.n1.a6

[9] Liang, H. \& Chen, X., Capillary-gravity ship wave patterns. Journal of Hydrodynamics, Ser. B, 29(5), pp. 825-830, 2017. https://doi.org/10.1016/s1001-6058(16)60794-1

[10] Gnitko, V., Degtyariov, K., Karaiev, A. \& Strelnikova, E., Multi-domain boundary element method for axisymmetric problems in potential theory and linear isotropic elasticity. WIT Transactions on Engineering Sciences, 122, WIT Press, pp.13-25, 2019. doi:10.2495/BE410021

[11] Rusanov, A., Rusanov, R. \& Lampart, P., Designing and updating the flow part of axial and radial-axial turbines through mathematical modelling. Open Engineering (formerly Central European Journal of Engineering), 5, pp. 399-410, 2015, doi:10.1515/eng2015-0047

[12] Brebbia, C.A., Telles, J.C.F. \& Wrobel, L.C., eds., Boundary Element Techniques, Springer-Verlag: Berlin and New York, pp. 338-357, 1984.

[13] Landau, L. D. \& Lifshitz, E. M., Fiuid Mechanics, 2nd ed., Pergamon, New York, pp. 238-250, 1987.

[14] Gnitko, V., Degtyariov, K., Karaiev, A. \& Strelnikova, E., Singular boundary method in a free vibration analysis of compound liquid-filled shells. WIT Transactions on Engineering Sciences, 126, WIT Press, pp. 189-200, 2019. doi:10.2495/BE420171

[15] Gnitko, V., Degtyariov, K., Naumenko, V., Strelnikova, E., BEM and FEM analysis of the fluid-structure Interaction in tanks with baffles. Int. Journal of Computational Methods and Experimental Measurements, 5(3), pp. 317-328, 2017. https://doi.org/10.2495/ cmem-v5-n3-317-328

[16] Gnitko, V., Degtyarev, K., Naumenko, V. \& Strelnikova, E., Coupled BEM and FEM analysis of fluid-structure interaction in dual compartment tanks. Int. Journal of Com- 
putational Methods and Experimental Measurements, 6(6), pp. 976-988, 2018. https:// doi.org/10.2495/cmem-v6-n6-976-988

[17] Karaiev, A. \& Strelnikova, E., Singular integrals in axisymmetric problems of elastostatics. Int. J. Model. Simul. Sci. Comput. 11(1), 2050003, 2020. doi:10.1142/ S1793962320500038

[18] Ibrahim, R. A., Liquid Sloshing Dynamics: Theory and Applications. Cambridge University Press, pp. 9-23, 2005.

[19] Gavrilyuk, I., Hermann, M., Lukovsky, I., Solodun, O. \& Timokha, A., Natural sloshing frequencies in truncated conical tanks. Engineering Computations, 25(6), pp. 518-540, 2008. https://doi.org/10.1108/02644400810891535

[20] Mciver, P., Sloshing frequencies for cylindrical and spherical containers filled to an arbitrary depth. J. Fluid Mech., 201, pp. 243-257, 1989. https://doi.org/10.1017/ s0022112089000923 